\title{
Caracterización de las áreas de sistema respiratorio en España
}

\author{
J. I. DE GRANDA-ORIVE, F. GARCÍA-RÍO'1, F. ROIG-VÁZQUEZ², \\ R. ALEIXANDRE-BENAVENT ${ }^{3}$, J. C. VALDERRAMA-ZURIAN ${ }^{3}$, \\ J. M. MARTÍNEZ-ALBIACH, L. CALLOL-SÁNCHEZ \\ Servicios de Neumología. Hospital Central de la Defensa Gómez Ulla. \\ ${ }^{1}$ Hospital Universitario La Paz. Madrid. ${ }^{2}$ Hospital General Básico de la Defensa. \\ ${ }^{3}$ Instituto de Historia de la Ciencia y Documentación López Piñero (CSIC - Universidad \\ de Valencia) Facultad de Medicina. Valencia
}

CHARACTERIZATION OF THE LEADERSHIP SUBJECT AREAS IN THE RESPIRATORY FIELD IN SPAIN

\section{RESUMEN}

Objetivo: Fue caracterizar las principales áreas temáticas en el sistema respiratorio a través de un estudio bibliométrico.

Material y método: Para identificar y caracterizar las diferentes áreas temáticas del sistema respiratorio en España, se recopilaron los trabajos del área empleando una revista que fuera representativa de un amplio número de investigadores. En este sentido la revista Archivos de Bronconeumología es la publicación más importante en este campo en lengua española.

Resultados: Fueron analizados un total de 2.198 artículos publicados en Archivos de Bronconeumología desde 1970 al año 2000. En cada década, no se encontraron diferencias significativas excepto en el índice de productividad en el área de oncología en la década de los ochenta, en las áreas de insuficiencia respiratoria y trastornos del sueño (IRTS) y oncología en la década de los noventa, además de en el índice de Price en el área de técnicas diagnósticas y terapéuticas en la década de los setenta. Al comparar el índice de productividad de cada área, entre décadas, hemos observado un incremento significativo en la década de los noventa con respecto a la de los setenta en las áreas de asma, IRTS, tuberculosis, infecciones no tuberculosas, circulación, oncología, enfermedades pleurales y enfermedades intersticiales, y en las áreas de asma, IRTS, infecciones no tuberculosas, circulación y enfermedades pleurales entre la década de los noventa y la de los ochenta. El área de tuberculosis mantiene el índice de aislamiento más alto, pero hemos encontrado un incremento progresivo en el mismo en las áreas de IRTS, infecciones no tuberculosas, oncología y enfermedades intersticiales.

Conclusiones: En general todos los indicadores se mantienen estables aunque las áreas más productivas fueron IRTS y oncología. La productividad aumento en las áreas de asma, IRTS, tuberculosis, infecciones no tuberculosas, oncología, circulación y enfermedades pleurales e intersticiales.

PALABRAS CLAVE: Bibliometría. Sistema respiratorio. España. Información.

\section{ABSTRACT}

Objective: The aim of this study was to characterize the leading topics in respiratory system in Spain through a bibliometric analysis.

Material and method: For identify and characterize the performance of the different research topics in respiratory system in Spain, we compile the production using a journal that turn out representative of a broad group of researcher. In this sense the journal Archivos de Bronconeumología is the most important publication in Spanish language of this field.

Results: A total of 2198 articles published in Archivos Bronconeumología from 1970 to 2000 were analyzed. In each three decades, we did not found differences except in the productivity index in oncology in the eighty decade and in respiratory failure and sleep disturbance (RFSD) and oncology areas in the ninetieth decade and in the Price index (consumption indicators) in the diagnostic and therapeutic techniques area in seventy decade. When we compare the productivity index of each subject areas between decades, we found a significant production increase in the ninetieth decade in asthma, RFSD, tuberculosis, non tuberculosis infection, circulation, oncology, pleural disease and interstitial areas versus the same in seventy decade, and also, we found significant differences between ninetieth and eighty decades in the asthma, RFSD, non tuberculosis infection, circulation and pleural disease areas. Tuberculosis area maintains an insularity index higher than the other areas. We also found a progressive increase in the insularity index of RFSD, non tuberculosis infection, oncology and interstitial disease areas.

Conclusions: In general all the indicators maintains stable although the more productivity topics were respiratory failure and sleep disturbances, and oncology. The productivity has increased in asthma, respiratory failure and sleep disturbances, tuberculosis, non - tuberculosis respiratory infections, oncology, pulmonary circulation, pleura and interstitial disease.

KEY WORDS: Bibliometric. Respiratory system. Spain. Research evaluation.

De Granda-Orive JI, García-Río F, Roig-Vázquez F, Aleixandre-Benavent R, Valderrama-Zurían JC, Martínez-Albiach JM, Callol-Sánchez L. Caracterización de las áreas de sistema respiratorio en España. An Med Interna (Madrid) 2006; 23: 513-518.

\section{INTRODUCCIÓN}

La importancia de la investigación en el progreso y desarrollo de las naciones es un hecho conocido. Además de la evidente repercusión sanitaria y socioeconómica, ha sido demostrado que la producción científica de la Unión Europea se ha incrementado considerablemente las últimas décadas (1-3).

Para caracterizar e identificar el rendimiento de las distintas áreas de investigación se pueden emplear diferentes estra-

Trabajo aceptado: 7 de junio de 2006 
tegias como analizar la distribución y el origen geográfico de la producción total, recopilar la producción mediante el empleo de palabras clave o valorar la distribución por áreas en una revista que resulte representativa de un amplio grupo de investigadores. En este sentido la revista Archivos de Bronconeumología, órgano de publicación oficial de la Sociedad Española de Neumología y Cirugía Torácica (SEPAR) y de la Asociación Latinoamericana del Tórax (ALAT), es la revista más importante en lengua española en este campo $(4,5)$.

La bibliometría tiene por objeto el tratamiento y estudio de datos cuantitativos procedentes de las publicaciones científicas, es decir, pretende cuantificar la actividad científica (6). El estudio de la producción, circulación, consumo y repercusión de las publicaciones, en definitiva, el comportamiento de la información científica compete a la bibliometría (7). En los estudios bibliométricos se distingue entre citas (que una publicación recibe de otras posteriores) y referencias (que una publicación hace de otras anteriores), y el objetivo del estudio de las citas y referencias es el consumo de información científica, y conocer la repercusión o impacto que su producción ha tenido en comunidades científicas determinadas $(8,9)$.

El objetivo de este estudio ha sido caracterizar las principales áreas temáticas del sistema respiratorio en España a través de un análisis bibliométrico.

\section{MATERIAL Y MÉTODOS}

\section{RECOPILACIÓN DE DATOS Y VARIABLES ANALIZADAS}

Revisión manual: Fueron seleccionados todos los números de Archivos de Bronconeumología publicados desde el año 1970 al 2000 (ambos incluidos). Las revistas monográficas, los suplementos y las que incluyen las ponencias y abstracts de los congresos no han sido incluidas en el estudio.

Las variables analizadas fueron el año de publicación, el área (asma bronquial, tabaquismo, insuficiencia respiratoria y trastornos del sueño (IRTS), técnicas diagnósticas y terapéuticas (TDT), tuberculosis, infecciones no tuberculosas, oncología, circulación pulmonar, pleura, fisiopatología y función pulmonar y enfermedades intersticiales), el tipo de documento (editorial, original, revisión, nota clínica y carta al director), número de autores, la institución de los autores, especialidad de los autores, el tiempo desde la recepción del artículo hasta su aceptación y desde su aceptación hasta su publicación, las palabras clave y el número y distribución de las referencias. Debido al número infinito de revistas que pueden aparecer en las referencias de los artículos publicados en Archivos de Bronconeumología, se han tomado únicamente las 24 publicaciones periódicas que previamente habían sido las más citadas en un estudio previo (10,11). Además, Archivos de Bronconeumología fue incluida para determinar la autocitación. Otras variables analizadas fueron el número de referencias por artículo y año desde 1970 a 2000, la autocitación de la revista, del primer autor y del grupo de trabajo.

\section{INDICADORES BIBLIOMÉTRICOS}

Como indicador de producción se calculó el índice de productividad (logaritmo del número de artículos), y además el índice de cooperación (número de autores por artículo), y el número de referencias por artículo. En cuanto a los indicadores de consumo fueron calculados el índice de obsolescencia (mediana de la distribución de las referencias), el índice de Price (porcentaje de referencias con menos de cinco años de antigüedad), el índice de aislamiento (porcentaje de referencias del mismo país que la publicación citadora), distribución de las referencias según la revista fuente, y el índice de autocitación (número de autocitaciones por artículo en Archivos de Bronconeumología)

\section{ANÁLISIS ESTADÍSTICO}

El análisis estadístico se realizó mediante el programa "Statistical Package for the Social Sciences" (SPSS), version 11.0. Los resultados fueron expresados como la media aritmética \pm la desviación estándar. El test del Chi-cuadrado fue utilizado para la comparación de proporciones y un modelo de distribución binomial se empleó para comparaciones de porcentajes grupo a grupo. El test de la t de Student fue empleado para la comparación de medias de dos grupos para una variable cuantitativa continua, después de confirmar una distribución normal con el test de Kolmogorov-Smirnov. La potencia de asociación entre dos variables fue medida con el test de correlación de Pearson. Para la comparación de una media cuantitativa continua para más de dos grupos se realizó un análisis de la varianza (ANOVA) con comparaciones post hoc por el test de Bonferroni. Se aceptó una $\mathrm{p}<0.05$ (IC 95\%).

\section{RESULTADOS}

\section{DESCRIPCIÓN GENERAL}

Fueron analizados un total de 2.198 artículos publicados en Archivos Bronconeumología desde 1970 a 2000 (ambos inclusive). Un 39,2\% de los documentos fueron artículos originales y un $29,6 \%$ cartas al director. Por nacionalidad de los autores, $2.134(97,1 \%)$ documentos lo fueron por autores españoles. Los autores extranjeros más productivos fueron de Holanda, Estados Unidos, y Argentina y Francia, con 10, 9, y 8 artículos respectivamente. Aunque existe una gran dispersión, en cuanto a la distribución de los artículos por provincia española, el $48 \%$ de los documentos vinieron de Barcelona, Madrid, y Valencia. La media $( \pm \mathrm{DE})$ del número de autores por artículo de todos los artículos publicados en el periodo estudiado fue de $4 \pm 2$ (rango: 1 a 18). El tiempo medio de aceptación de los artículos fue de $104 \pm 84$ días (rango: 1 a 683 días) y la media del tiempo medio desde la aceptación hasta la publicación del artículo fue de $167 \pm 55$ días (rango: 9 a 395 días). La media del número de referencias por artículo fue de $18 \pm 20$.

Aunque la especialidad de los autores que publica en Archivos de Bronconeumología pertenece a 42 especialidades diferentes ( 88 de ellos no indicaron su especialidad), existe un claro predominio de artículos firmados por neumólogos (1460 documentos, $49 \%$ ), siendo el segundo y el tercer puesto para cirugía torácica y medicina interna, con $402(13,5 \%)$ y 246 artículos $(8,3 \%)$, respectivamente. La distribución de artículos por institución de los autores también presenta una gran dispersión, pero en general los hospitales y las universidades son los contribuyentes más frecuentes. 


\section{CARACTERIZACIÓN DE LAS PRINCIPALES ÁREAS TEMÁTICAS}

Identificamos artículos de todas las áreas. El índice de cooperación se mantuvo entre 3 y 5 autores y los intervalos de aceptación y publicación no variaron significativamente en todas las áreas y décadas.

En la tabla I se muestra la evolución del índice de productividad por cada área entre décadas. En general por área, en cada década, no hemos encontrado diferencias significativas excepto en el índice de productividad del área de oncología en la década de los ochenta y en el área de IRTS y oncología en la década de los noventa. Cuando se comparó el índice de productividad de cada área entre décadas, encontramos un incremento significativo de la producción en la década de los noventa con respecto a la de los setenta en las áreas de asma, IRTS, tuberculosis, infecciones no tuberculosas, circulación, oncología, enfermedades pleurales e intersticiales y en las áreas de asma, IRTS, infecciones no tuberculosas, circulación y enfermedades pleurales al compararla década de los noventa con la década de los ochenta.

En la tabla II se muestra la evolución de los indicadores de consumo para cada área. En general no encontramos en cada década, para cada área, diferencias en ninguno de los indicadores excepto en el índice de Price en el área de TDT en la década de los setenta. El área de tuberculosis mantiene un índice de aislamiento mayor que el del resto de áreas. De todas maneras encontramos igualmente un incremento progresivo del índice de aislamiento en las áreas de IRTS, infecciones no tuberculosas, oncología y enfermedades intersticiales.

En la tabla III se pueden observar las autocitaciones para cada área temática. El área de tuberculosis es la que presenta un índice mayor de autocitación de artículos, y por autor y en cambio, por grupo de trabajo, destaca el área de asma.

\section{DISCUSIÓN}

En primer lugar, somos conscientes de que, desde un punto de vista general nuestro trabajo presenta una serie de limita- ciones metodológicas, como son la fuente de adquisición de datos y los indicadores bibliométricos (7). Nuestro estudio emplea los datos de una revista, pero por el momento dicha publicación es la más importante en lengua española en el sistema respiratorio. Por supuesto que el sistema respiratorio se incluye en una variedad de disciplinas; muchos artículos de investigación básica respiratoria son publicados en revistas de ciencia básica, y ensayos clínicos de respiratorio aparecen en otras categorías cubiertas por áreas generales de la ciencia. La decisión de realizar el estudio sólo en una revista se tomó en base a que pensamos que el análisis tiene consistencia, empleando un método sencillo y repetible y pudiendo así, con nuestra revista, ser evaluada la evolución en el tiempo del sistema respiratorio en España.

La evaluación de la investigación es en la actualidad el foco de un debate sustancial en todo el mundo por la necesidad de otorgar los recursos financieros y materiales disponibles. Por ello, se hace necesario evaluar la actividad investigadora del sistema respiratorio, no solo para conocer la producción científica de un país, con el fin de otorgar recursos, sino por lo que implica de beneficio a la comunidad, siendo una fuente de información importante. Dicha evaluación permite al país definir su posición con respecto a sus competidores y puede así explotar mejor las oportunidades en todos los campos de la ciencia (12).

Según Camí y cols. (13), España ocupa el undécimo puesto en producción biomédica a nivel mundial, y el séptimo en la Unión Europea (según otros autores el sexto) (14). El problema es que si la contribución española en cuanto a producción biomédica a nivel mundial es del $2,4 \%$ este porcentaje baja al $1,8 \%$ en cuanto a número de citaciones (13), estando por debajo de la media internacional.

La importancia del sistema respiratorio ha aumentado en los últimos años, encontrándose un incremento en la repercusión del sistema, incluso observado a través del Journal Citation Report (JCR) que sólo incluía 30 revistas en ésta categoría. Si estudiamos la producción española en sistema respiratorio desde el año 1994 al 2000, se puede observar que

TABLA I

EVOLUCIÓN DEL ÍNDICE DE PRODUCTIVIDAD PARA CADA ÁREA ENTRE DÉCADAS*

\begin{tabular}{lcccc}
\hline Área & $1970-1979$ & $1980-1989$ & $1990-1199$ & TOTAL \\
\hline Asma & $0,18 \pm 0,16^{\mathrm{a}}$ & $0,40 \pm 0,30^{\mathrm{a}}$ & $0,86 \pm 0,18$ & $0,54 \pm 0,36$ \\
Tabaco & - & 0,30 & $0,43 \pm 0,37$ & - \\
IRTS & $0,51 \pm 0,19^{\mathrm{a}}$ & $0,52 \pm 0,32^{\mathrm{a}}$ & $1,11 \pm 0,21$ & $0,73 \pm 0,38$ \\
TDT & $0,55 \pm 0,16$ & $0,55 \pm 0,28$ & $0,69 \pm 0,38$ & $0,61 \pm 0,30$ \\
TB & $0,23 \pm 0,20^{\mathrm{b}}$ & $0,40 \pm 0,35$ & $0,65 \pm 0,33$ & $0,44 \pm 0,34$ \\
INT & $0,32 \pm 0,28^{\mathrm{a}, \mathrm{c}}$ & $0,76 \pm 0,20^{\mathrm{b}}$ & $1,05 \pm 0,22$ & $0,77 \pm 0,35$ \\
Oncología & $0,63 \pm 0,34^{\mathrm{a}, \mathrm{c}}$ & $1,10 \pm 0,21$ & $1,26 \pm 0,17$ & $1,05 \pm 0,33$ \\
Circulación & $0,60 \pm 0,21^{\mathrm{b}}$ & $0,40 \pm 0,38$ & $0,63 \pm 0,32$ & $0,43 \pm 0,37$ \\
Pleura & $0,48 \pm 0,25^{\mathrm{a}}$ & $0,61 \pm 0,30^{\mathrm{b}}$ & $1,04 \pm 0,15$ & $0,75 \pm 0,33$ \\
PFuncionales & $0,41 \pm 0,26$ & $0,42 \pm 0,26$ & $0,64 \pm 0,13$ & $0,49 \pm 0,24$ \\
Intersticial & $0,5 \pm 0,28^{\mathrm{a}, \mathrm{c}}$ & $0,83 \pm 0,29$ & $1,01 \pm 0,18$ & $0,79 \pm 0,33$ \\
\hline
\end{tabular}

IRTS: insuficiencia respiratoria y trastornos del sueño; TDT: técnicas diagnósticas y terapéuticas; TB: tuberculosis; INT: infecciones no tuberculosas; PFuncionales: pruebas de función pulmonar. * Los valores son la media \pm DE

Comparación entre décadas de cada área: ${ }^{a} p<0,001$ vs. 1990-1999. ${ }^{b} p<0,05$ vs. 1990-1999. ${ }^{\mathrm{c}} \mathrm{p}<0,05$ vs. $1980-1989$. 
TABLA II

EVOLUCIÓN DE LOS INDICADORES DE CONSUMO PARA CADA ÁREA*

\begin{tabular}{|c|c|c|c|c|c|}
\hline & Décadas & Referencias & Índice de obsolescencia & Índice Price & Índice aislamiento \\
\hline Asma & $\begin{array}{c}70-79 \\
80-89 \\
90-99 \\
\text { Total }\end{array}$ & $\begin{array}{c}13 \pm 4 \\
13 \pm 3 \\
29 \pm 12 \\
20 \pm 11\end{array}$ & $\begin{array}{l}5,7 \pm 0,8 \\
5,4 \pm 1,7 \\
6,0 \pm 1,3 \\
5,7 \pm 1,4\end{array}$ & $\begin{array}{c}41,9 \pm 9,6 \\
45,9 \pm 16,0 \\
43,0 \pm 9,2 \\
43,9 \pm 12,0\end{array}$ & $\begin{array}{l}5,4 \pm 6,5 \\
2,5 \pm 2,8 \\
4,2 \pm 2,8 \\
3,8 \pm 3,8\end{array}$ \\
\hline Tabaco & $\begin{array}{l}70-79 \\
80-89 \\
90-99\end{array}$ & $\begin{array}{c}- \\
29 \\
26 \pm 10\end{array}$ & $\begin{array}{c}- \\
2,1 \\
5,1 \pm 3,4\end{array}$ & $\begin{array}{c}- \\
75 \\
53,1 \pm 18,7\end{array}$ & $\begin{array}{c}- \\
3,4 \\
10,6 \pm 9,2\end{array}$ \\
\hline IRTS & $\begin{array}{c}70-79 \\
80-89 \\
90-99 \\
\text { Total }\end{array}$ & $\begin{array}{c}18 \pm 7 \\
23 \pm 10 \\
20 \pm 6 \\
21 \pm 8\end{array}$ & $\begin{array}{l}6,3 \pm 1,9 \\
6,4 \pm 2,1 \\
6,4 \pm 1,1 \\
6,4 \pm 1,6\end{array}$ & $\begin{array}{c}33,6 \pm 9,5 \\
35,7 \pm 18,7 \\
39,9 \pm 7,0 \\
37,0 \pm 13,1\end{array}$ & $\begin{array}{l}3,6 \pm 3,7 \\
2,0 \pm 2,0 \\
7,4 \pm 3,1 \\
4,4 \pm 3,7\end{array}$ \\
\hline TDT & $\begin{array}{c}70-79 \\
80-89 \\
90-99 \\
\text { Total }\end{array}$ & $\begin{array}{c}12 \pm 4 \\
11 \pm 5 \\
30 \pm 23 \\
19 \pm 17\end{array}$ & $\begin{array}{l}3,2 \pm 0,4 \\
6,9 \pm 2,4 \\
5,5 \pm 2,2 \\
5,8 \pm 2,4\end{array}$ & $\begin{array}{l}69,7 \pm 23,4 \\
39,4 \pm 13,6 \\
50,2 \pm 14,4 \\
49,8 \pm 19,2\end{array}$ & $\begin{array}{l}5,1 \pm 4,4 \\
6,9 \pm 5,5 \\
3,7 \pm 2,7 \\
5,3 \pm 4,4\end{array}$ \\
\hline TB & $\begin{array}{c}70-79 \\
80-89 \\
90-99 \\
\text { Total }\end{array}$ & $\begin{array}{l}23 \pm 8 \\
15 \pm 7 \\
19 \pm 8 \\
19 \pm 8\end{array}$ & $\begin{array}{l}4,4 \pm 0,5 \\
5,4 \pm 1,1 \\
5,2 \pm 1,7 \\
5,2 \pm 1,4\end{array}$ & $\begin{array}{l}49,6 \pm 20,5 \\
45,1 \pm 14,8 \\
50,7 \pm 14,0 \\
42,3 \pm 15,5\end{array}$ & $\begin{array}{c}10,3 \pm 18,7 \\
6,1 \pm 6,5 \\
19,0 \pm 9,7 \\
11,9 \pm 12,9\end{array}$ \\
\hline INT & $\begin{array}{c}70-79 \\
80-89 \\
90-99 \\
\text { Total }\end{array}$ & $\begin{array}{l}13 \pm 3 \\
17 \pm 7 \\
22 \pm 9 \\
18 \pm 8\end{array}$ & $\begin{array}{l}5,3 \pm 1,4 \\
6,6 \pm 1,7 \\
6,0 \pm 1,4 \\
6,2 \pm 1,5\end{array}$ & $\begin{array}{c}31,2 \pm 26,7 \\
37,6 \pm 9,3 \\
43,7 \pm 9,6 \\
38,7 \pm 14,4\end{array}$ & $\begin{array}{l}1,3 \pm 2,2 \\
7,2 \pm 5,2 \\
6,8 \pm 3,4 \\
5,7 \pm 4,6\end{array}$ \\
\hline Oncología & $\begin{array}{c}70-79 \\
80-89 \\
90-99 \\
\text { Total }\end{array}$ & $\begin{array}{l}16 \pm 9 \\
18 \pm 4 \\
15 \pm 4 \\
16 \pm 5\end{array}$ & $\begin{array}{l}6,9 \pm 6,4 \\
6,9 \pm 1,1 \\
6,2 \pm 1,8 \\
6,6 \pm 1,4\end{array}$ & $\begin{array}{c}32,6 \pm 7,2 \\
35,2 \pm 6,8 \\
42,4 \pm 10,2 \\
37,5 \pm 9,1\end{array}$ & $\begin{array}{l}2,9 \pm 3,5 \\
6,6 \pm 2,7 \\
6,1 \pm 1,3 \\
5,5 \pm 2,8\end{array}$ \\
\hline Circulación & $\begin{array}{c}70-79 \\
80-89 \\
90-99 \\
\text { Total }\end{array}$ & $\begin{array}{l}16 \pm 5 \\
16 \pm 6 \\
13 \pm 4 \\
15 \pm 5\end{array}$ & $\begin{array}{c}5,5 \\
7,1 \pm 2,0 \\
6,8 \pm 2,6 \\
6,8 \pm 2,3\end{array}$ & $\begin{array}{l}24,1 \pm 16,0 \\
29,6 \pm 13,7 \\
39,5 \pm 15,5 \\
33,3 \pm 15,6\end{array}$ & $\begin{array}{l}2,7 \pm 4,8 \\
1,8 \pm 2,4 \\
5,5 \pm 3,6 \\
3,7 \pm 3,8\end{array}$ \\
\hline Pleura & $\begin{array}{c}70-79 \\
80-89 \\
90-99 \\
\text { Total }\end{array}$ & $\begin{array}{l}14 \pm 4 \\
18 \pm 8 \\
12 \pm 3 \\
15 \pm 6\end{array}$ & $\begin{array}{c}6,7 \\
8,3 \pm 1,2 \\
6,3 \pm 1,9 \\
7,2 \pm 1,8\end{array}$ & $\begin{array}{c}31,9 \pm 7,0 \\
30,8 \pm 7,9 \\
41,7 \pm 11,9 \\
35,4 \pm 10,6\end{array}$ & $\begin{array}{c}7,3 \pm 6,9 \\
8,0 \pm 4,2 \\
10,4 \pm 2,2 \\
8,8 \pm 4,4\end{array}$ \\
\hline Funcionales & $\begin{array}{c}70-79 \\
80-89 \\
90-99 \\
\text { Total }\end{array}$ & $\begin{array}{c}13 \pm 6 \\
20 \pm 22 \\
29 \pm 17 \\
21 \pm 18\end{array}$ & $\begin{array}{l}5,3 \pm 1,8 \\
7,9 \pm 2,9 \\
7,4 \pm 3,5 \\
7,4 \pm 3,1\end{array}$ & $\begin{array}{l}41,1 \pm 11,4 \\
28,1 \pm 17,3 \\
38,7 \pm 15,9 \\
34,8 \pm 16,2\end{array}$ & $\begin{array}{c}2,6 \pm 4,5 \\
5,6 \pm 10,0 \\
4,8 \pm 5,5 \\
4,5 \pm 7,1\end{array}$ \\
\hline Intersticial & $\begin{array}{c}70-79 \\
80-89 \\
90-99 \\
\text { Total }\end{array}$ & $\begin{array}{l}19 \pm 9 \\
16 \pm 5 \\
15 \pm 7 \\
17 \pm 7\end{array}$ & $\begin{array}{l}6,4 \pm 2,4 \\
7,4 \pm 2,1 \\
6,7 \pm 1,8 \\
6,9 \pm 1,9\end{array}$ & $\begin{array}{c}34,9 \pm 12 \\
35,6 \pm 8,8 \\
37,9 \pm 12,5 \\
36,4 \pm 10,6\end{array}$ & $\begin{array}{c}2,72 \pm 2,3 \\
5,2 \pm 3,1 \\
7,26 \pm 2,8 \\
5,23 \pm 3,2\end{array}$ \\
\hline
\end{tabular}

IRTS: insuficiencia respiratoria y trastornos del sueño; TDT: técnicas diagnósticas y terapéuticas; TB: tuberculosis; INT: infecciones no tuberculosas; Funcionales: pruebas funcionales respiratorias. ${ }^{*}$ Los valores son la media $\pm \mathrm{DE}$. 
TABLA III

\begin{tabular}{lccc} 
& & TABLA III & \\
& Autocitación revista & Autocitación autor & Autocitación grupo \\
\hline Asma & $2,32 \pm 3,03$ & $3,08 \pm 2,5$ & $5,42 \pm 4$ \\
Tabaco & $1,63 \pm 1,21$ & $1,48 \pm 1,31$ & $2,67 \pm 2,51$ \\
IRTS & $2,92 \pm 3,16$ & $2,19 \pm 3,37$ & $3,9 \pm 4,17$ \\
TDT & $4,6 \pm 4,19$ & $1,42 \pm 2,04$ & $3,59 \pm 6,28$ \\
TB & $5,04 \pm 7,27$ & $2,79 \pm 4,24$ & $4,54 \pm 4,27$ \\
INT & $2,04 \pm 2,62$ & $0,82 \pm 1,31$ & $1,73 \pm 2,11$ \\
Oncología & $2,98 \pm 2,02$ & $0,94 \pm 0,93$ & $1,73 \pm 1,45$ \\
Circulación & $2,11 \pm 2,91$ & $0,29 \pm 0,80$ & $0,68 \pm 1,59$ \\
Pleura & $4,81 \pm 3,75$ & $2,39 \pm 2,92$ & $3,09 \pm 3,33$ \\
Funcionales & $3,75 \pm 6,74$ & $2,19 \pm 2,6$ & $4,73 \pm 5,25$ \\
Intersticiales & $2,95 \pm 2,07$ & $1,42 \pm 1,84$ & $2,68 \pm 2,6$ \\
\hline
\end{tabular}

IRTS: insuficiencia respiratoria y trastornos del sueño; TDT: técnicas diagnósticas y terapéuticas; TB: tuberculosis; INT: infecciones no tuberculosas; Funcionales: pruebas funcionales respiratorias. ${ }^{*}$ Los valores son la media $\pm \mathrm{DE}$.

ha sido una de las disciplinas con más de 1.000 documentos citables $(2,13)$. La tendencia actual del sistema respiratorio es ascendente, colocándose en la actualidad en el lugar número 15 en cuanto a productividad $(2,13,15)$ en nuestro país.

En este punto la pregunta que nos podemos hacer es ¿existen diferencias en los indicadores de productividad entre las diferentes áreas de respiratorio? En este trabajo hemos encontrado que las áreas más productivas han sido IRTS y oncología. La importancia del área de oncología es innegable, un reciente estudio analizaba los artículos incluídos en el JCR en el período 1996 a 2000 (12), encontrando un total de 66.021 documentos del área de oncología en todo el mundo, siendo el $35,5 \%$ de ellos de autores de la Unión Europea. El país más productivo fue el Reino Unido (UK) (20,3\%), seguido de Italia $(18,1 \%)$, Alemania $(15,2 \%)$, Francia $(12,7 \%)$ y los Países Bajos (9,1\%). España, con el 3,5\% del total de documentos, incremento su producción de 117 artículos en 1995 a 817 en el año 2000, con un factor de impacto medio de 2,8 (2,1 en 1995 a 3,1 en el año 2000). Al ordenar la producción de documentos, relativizándola con el número de habitantes o por el producto interior bruto, España empeora su situación en el ranking (12). En otro estudio (16), que sitúa la producción de investigación en cáncer de acuerdo con las publicaciones sobre el tema (búsqueda realizada en la base de datos MEDLI$\mathrm{NE}$ ) encuentra que entonces Estados Unidos se coloca en primer lugar en cuanto a producción, seguido de Italia y después Japón. España ocuparía el noveno puesto por número de documentos y la octava posición por repercusión.

Rippon y cols. (17), analizaron la producción científica en sistema respiratorio en todo el mundo en el período 1996 a 2001. Recopilaron un total de 81.419 documentos, empleando el Science Citation Index, y encontraron que Finlandia, Canadá, España y el Reino Unido son países con cierta importancia en sistema respiratorio. Además los autores subdividieron la producción en sistema respiratorio en catorce áreas temáticas. Encontraron que tras el área miscelanea, eran las áreas de asma, cáncer de pulmón y las enfermedades pediátricas pulmonares las que recopilaban la mayor producción. En concordancia con los hallazgos encontrados por nosotros en este trabajo, España es particularmente productiva en el tema de investigación en las manifestaciones pulmonares del HIV, así como en los campos temáticos de cáncer, IRTS, tuberculosis o neumonías (17).
Estamos de acuerdo con los autores en que estos estudios que analizan la producción en diferentes áreas de la ciencia encuentran que existen variaciones en los logros en la investigación en sistema respiratorio y ello no siempre depende del daño producido por dichas enfermedades en cada nación.

Recientemente, Michalopoulus y cols. (18), en un trabajo en el que analizaban la contribución de las diferentes regiones del mundo en investigación en sistema respiratorio, a través de la base de datos PubMed, en el período 1995 a 2003 y sobre 30 revistas, que tienen la categoría de pertenecer al sistema respiratorio según el JCR, recopilaron un total de 49.382 documentos (originales y revisiones), siendo la mayoría de autores de la Europa del oeste $(40,4 \%)$ y de Estados Unidos $(35,4 \%)$, aunque relativizando por el número de habitantes o la renta per - capita, destacaban Canadá y Oceanía. Los autores concluyen que la producción científica en sistema respiratorio mantiene una tendencia ascendente en el periodo estudiado, aunque no es homogénea en todo el mundo, siendo la Europa occidental y Estados Unidos los más productivos en números absolutos, y Canadá y Oceanía al relativizar al producción por número de habitantes o renta per capita.

Son pocos los trabajos que evalúan la importancia y el impacto de la investigación biomédica en Europa. Lewison y cols. (19) en un trabajo en el que analizaban el volumen y el impacto potencial de la investigación en gastroenterología, concluyen de la importancia de la Unión Europea en este campo, habiéndose encontrado lo mismo en otros estudios de distintas disciplinas como cuidados intensivos (20), anestesia (21), radiología $(22)$, oncología $(12,16)$ y enfermedades infecciosas (23), y por supuesto en sistema respiratorio $(1,17,18)$. En todos los campos se ha observado que la Unión Europea ha incrementado no sólo su producción sino también su repercusión, no siendo diferente en el sistema respiratorio $(24,25)$.

Podemos concluir que en general todos los indicadores se han mantenido estables en todas las áreas del sistema respiratorio, sin grandes diferencias entre ellos. Aunque las más productivas fueron las áreas de IRTS y de oncología, cuando comparamos la evolución de los indicadores de producción de cada área entre décadas, no observamos grandes diferencias, pero la productividad se ha incrementado en las áreas de asma, IRTS, tuberculosis, infecciones no tuberculosas, oncología, circulación, pleura y enfermedades intersticiales. 


\section{Bibliografía}

1. García Río F, Serrano S, Dorgham A, Álvarez-Sala R, Ruiz Peña A, Pino JM, et al. A bibliometrics evaluation of European Union research of the respiratory system from 1987-1998. Eur Respir J 2001; 17: 117580 .

2. Camí J, Suñen E, Carbó JM, Coma L. Producción científica española en biomedicina y ciencias de la salud (1994-2000). Informe del Instituto de Salud Carlos III-Fondo de Investigación Sanitaria. Available in URL: http://www.isciii.es/fis/mapa/index.htm

3. Granda Orive JI, García Río F, Gutiérrez Jiménez T, Escobar Sacristán J, Riera Palmero J, Callol Sánchez L. Evolution of bibliometric indicators and his websites evaluation approaches in relation to the foremost respiratory journal in spanish. Cybermetrics 2004; 8 (1): Paper1. Available in URL: http://www.cindoc.csic.es/cybermetrics/articles/v8ilp1.html

4. García F, Mayoralas S, Dorgham A, Granda JI, Perpiña M, Casan P, et al. Análisis de repercusión de Archivos de Bronconeumología a través del Science Citation Index. Arch Bronconeumol 2001; 37: 465-70.

5. Perpiña Torderá M, Xaubet Mir A, Casán Clará P, García Río F, Sánchez de León R. Primer factor de impacto de Archivos de Bronconeumología. Arch Bronconeumol 2004; 40: 337.

6. Glänzel W. Bibliometrics as a research field. A course on theory and application of bibliometric indicators. (Course Handouts) 2003. Available in URL: http://www.norslis.net/2004/Bib_Module_KUL.pdf

7. González de Dios J, Moya M. Indicadores bibliométricos: características y limitaciones en el análisis de la actividad científica. An Esp Pediatr 1997; 47: 235-244.

8. López Piñero JM, Terrada ML. Los indicadores bibliométricos y la evaluación de la actividad científica (III). Los indicadores de producción, circulación, dispersión, consumo de la información y repercusión. Med Clin (Barc) 1992; 98: 142-48.

9. Granda Orive JI. Algunas reflexiones y consideraciones sobre el factor de impacto. Arch Bronconeumol 2003; 39: 409-17.

10. García Río F, Serrano S, Álvarez-Sala R, García Tejero T, Pino García JM, Álvarez-Sala JL, Villamor León J. Indicadores bibliométricos de producción y consumo de Archivos de Bronconeumología. Arch Bronconeumol 1996; 32: 327-31.

11. García Río F, Dorgham S, Serrano S, Terreros Caro FJ, Álvarez-Sala R, Prados C, et al. Evolución de los indicadores bibliométricos de producción y consumo de Archivos de Bronconeumología durante los últimos
20 años. Arch Bronconeumol 1997; 33: 20-6.

12. Ugolini D, Mela GS. Oncological research overview in the European Union. A 5-year survey. Eur J Cancer 2003; 39: 1888-94.

13. Cami J, Suñen Piñol E, Méndez Vasquez R. Mapa Bibliométrico de España 1994-2002: biomedicina y ciencias de la salud. Med Clin (Barc) 2005; 124: 93-101.

14. Gómez Caridad I, Fernández Muñoz T, Bordons Gangas M, Morillo Ariza F. La producción científica española en Medicina en los últimos años 1994-1999. Rev Clin Esp 2004; 204: 75-88.

15. Cami J, Suñen Piñol E, Méndez Vasquez R. Mapa Bibliométrico de España 1994-2002: biomedicina y ciencias de la salud. Informe del Instituto de Salud Carlos III-Fondo de Investigación Sanitaria. Available in URL: http://www.isciii.es/mapabiomedico.

16. Grossi F, Belvedere O, Rosso R. Geography of clinical cancer research publications from 1995 to 1999. Eur J Cancer 2003; 39: 106-11.

17. Rippon I, Lewison G, Partridge MR. Research outputs in respiratory medicine. Thorax 2005; 60: 63-7.

18. Michalopoulus A, Falagas ME. A bibliometric analysis of global research production in respiratory medicine. Chest 2005; 128: 3993-98.

19. Lewison G, Grant J, Jansen P. International gastroenterology research: subject areas, impact, and funding. Gut 2001; 49: 295-302.

20. Latronico N, Botteri M, Minelli C, Zanoti C, Bertolini G, Candiani A. Quality of reporting of randomised controlled trials in the intensive care literature. Int Care Med 2002; 28: 1316-23.

21. Figueredo E, Sánchez Perales G, Muñoz Blanco F. Internacional publishing in anaesthesia-how do different countries contribute? Acta Anaesthesiol Scand 2003; 47: 378-82

22. Mela GS, Martionoli C, Poggi E, Derchi LE. Radiological research in Europe: a bibliometric study. Eur Radiol 2003; 13: 657-662.

23. Ramos JM, Gutiérrez F, Masía M, Martín Hidalgo A. Publication of European Union research of infectious diseases (1991-2001): a bibliometric evaluation. Eur J Clin Microbiol Infect Dis 2004; 23: 180-84.

24. Sterk PJ, Rabe KF. Serving researchers, the impact factor and other conflicts of interest. Eur Respir J 2005; 25: 3-5.

25. García Río F, Serrano F, Álvaro D, Ruiz Manzano J, Dorgham A, Xaubet A, et al. Estimación de los indicadores bibliométricos de repercusión de Archivos de Bronconeumología. Arch Bronconeumol 1998; 34: 53135 . 